\title{
Realization of Seamless Mobility in Heterogeneous Wireless Networks based on IEEE 802.21 Framework
}

\author{
Vimal Kumar \\ Galgotias University \\ Plot\#2, Sector 17-A Taj Expressway \\ Greater Noida Gautam Budh Nagar(UP)-201308 India
}

\author{
Neeraj Tyagi \\ Motilal Nehru National Institute of Technology \\ Allahabad (UP) India
}

\author{
Ranjan Baghel \\ Moradabad Institute of Technology \\ Moradabad (UP) India
}

\begin{abstract}
Wireless and mobile networks are evolving very rapidly. The mobile nodes in the wireless networks are having multiple interfaces with different radio access technologies (RATs) which are having different capabilities, cost and performance ratio. The use of non-PC based portable devices is increasing due to their flexible usage. The wireless and mobile network which is formed by these non-PC and PC based devices is heterogeneous in nature and these networks are co-located. Multiple interfaces can be included in the mobile device by using separate hardware and software modules. A mobile user wants to be Always Best Connected (ABC) as per its various requirements and availability in a particular environment. When a mobile node leaves the current network and joins the other network, a handover operation is needed and performed. The Handover operation is used to achieve seamless mobility and is of two types, first is in between same RATs and second is in between different RATs. For seamless and smooth handover operations across heterogeneous networks, IEEE has published a standard, named as IEEE 802.21. This paper presents a comprehensive description on the issues and challenges for achieving the seamless mobility in a heterogeneous environment. Apart from this, we also present the description of services provided by IEEE 802.21 standard and related vertical handover schemes to realize seamless mobility in heterogeneous network.
\end{abstract}

\section{General Terms:}

Seamless Mobility, Vertical Handover

\section{Keywords:}

Multiple Interfaces, RATs, Heterogeneous Network, ABC, IEEE 802.21

\section{INTRODUCTION}

Now a days mobile node (MN) is evolving with multiple network interfaces and $\mathrm{MN}$ can have more than interface at the same time.
Along with this, different wireless technologies (such as cellular: $2 \mathrm{G}, 3 \mathrm{G}$ and $4 \mathrm{G}$, project 802: WLAN, WMAN etc.) are also evolving. These contemporary evolutionary development is forming the heterogeneous environment and can create the need of the availability of IP services anywhere at anytime on any network. The different networks in this heterogeneous environment have different RATs that may differ in terms of bandwidth, monetary cost, latency etc.

To decide the candidate point of attachment (PoA) for the MN, handover management is done for controlled switching of networks during ongoing active communication session[2]. The main issues involved with handover management are mobility scenarios, the parameters for handover and decision making algorithms. On the basis of movement of the MN among different types of networks, the mobility scenarios are of two types: horizontal and vertical. In horizontal mobility scenario, the MN roams around the different cells of the same network. In horizontal mobility scenario, horizontal handover is needed when the coverage of serving access point (AP) becomes unavailable because of switching among different cells of the same network. In vertical mobility scenario, the MN roams around different types of networks. In heterogeneous mobility scenario, vertical handover is required in between heterogeneous networks because of different radio access technology (RATs) used and different formats of link layer interfaces.

While handling the handover, the major challenges are: to decide the appropriate time to initiate the handover, to maintain the service continuity, to decide the candidate PoA, to implement automatic switching of the network interfaces and finally to achieve seamless mechanisms [2]. In case of the same RAT,the handover is called homogeneous handover. Homogeneous (horizontal) handover is already realized and less challenging due to the same RATs (serving RAT and candidate RAT).The heterogeneous (vertical) handover is more challenging and demanding. The vertical handover is not only initiated because of the connectivity reasons but also to provide convenience for the user. The vertical handover gives the options to the user for being always best connected (ABC) specially when the $\mathrm{MN}$ is equipped with multiple interfaces.The objective of vertical handover is to reduce the signaling overhead, eliminating the redundant handovers, and satisfying the network and user requirements. 


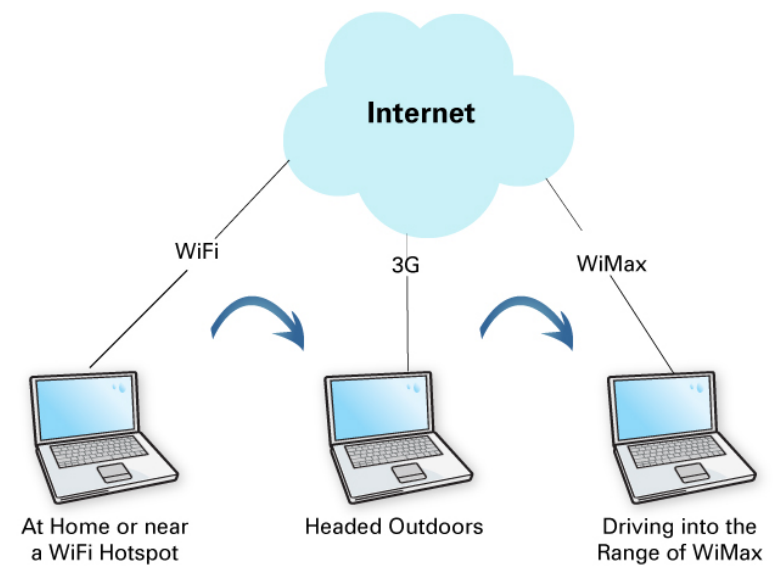

Fig. 1. Seamless Mobility in Wi-fi, 3G, WiMax

Ideally, a mobile user always does have a preference to be remain connected even if the user is on the move and the device is leaving one network and joining other network. The desperate and obsessed Internet users are always looking for mobility freedom. This requirement of mobile users is motivating the researchers and Industry to define, formulate and realize the Seamless mobility. Mobility management in such environment is a real challenge to achieve seamless mobility.

Seamless Mobility can be defined as the movement of a mobile node (MN) without interruption of the ongoing connection regardless of wherever the MN is. This is shown in the figure 1 . The realization of seamless mobility will also benefit for the mobile users in terms of cost and quality trade-off. To provide seamless mobility, a media independence service layer has to be developed and deployed in between the link layer and higher layers. To do this, we have to consider the compatibility issues among signaling schemes of different types of radio access technologies (RATs). There are basically the following issues with respect to seamless mobility[13,5]:

(1) Keeping the connection of the mobile node (MN) intact while it is moving

(2) Detecting the different network types

(3) Selecting the most appropriate network type based on the need, environment and services offered.

(4) Network Discovery

(5) Network selection and routing and connection update procedures

(6) Initiating handover operation

(7) Executing handover operation

The considered network technologies may include both wired, wireless and cellular technologies which are given in table 1 .

These all network technologies are converged and a common billing procedure is developed. Seamless mobility can be achieved by either implementing the component somewhere in the network or it is implemented in the mobile node(MN). Network-controlled seamless mobility method is prominent then MN-controlled, because in network-controlled seamless mobility method, load information of the cell and network status can be considered to achieve
Table 1.

RATs

\begin{tabular}{|l|l|l|}
\hline S.No & Network Technology Category & RATs \\
\hline 1. & Wired & Ethernet,Gigabit Ethernet \\
\hline 2. & Wireless & Wi-fi,Wi-MAX,Blue-tooth, \\
\hline 3. & Cellular & $2.5 \mathrm{G}$ to $4 \mathrm{G}$ \\
\hline 4. & Wired-cum- Wireless & DVB \\
\hline
\end{tabular}

load balancing and effective utilization of the available resources. The key challenge before the standardization bodies for the development of next generation networks (NGN) to provide seamless mobility is to focus on three mobility aspects:To provide seamless horizontal handover between same RATs, To provide seamless vertical handover between different RATs, Integration of different networks and RATs under same IP backbone, Maintaining the required QoS and security while switching among different RATs. The rest of this paper is organized as follows. First we described the mobility management at IP layer and application layer. Next we described media independent handover framework published by IEEE 802.21 working group.Next, we described network selection based on service zone for macro-mobility. Next we discussed the vertical handover management in which we described the issues and challenges to realize seamless mobility. Next We presented the simulation of vertical handover between WLAN and UMTS and various observations in tabular format. Finally the paper is concluded.

\section{MOBILITY MANAGEMENT AT IP LAYER}

Mobile IP provides the fundamental methods for mobility management at IP layer. As it is already described that mobility related functionality is either implemented in $\mathrm{MN}$ or somewhere in the network entity. This method is based on the concept of allocating two addresses for the MN. These two addresses helps in keeping the TCP connection intact while MN is visiting the foreign networks. In the two addresses assigned to the MN, one is the global address(permanent address) and other is the care of address (COA). The global address is the address of the MN in its home network and the COA is the address of $\mathrm{MN}$ in its current network which is called its foreign network. When the COA is obtained by the FA advertisements, it is called the FA-COA and when MN ob- 
tains it by using some external mechanism (DHCP), it is called the collocated-COA. When MN moves from its home network to the other network, first it has to inform its home network about its current location via an entity called the home agent (HA). Then HA registers the current address (COA), and whenever in the future the packet comes to the $\mathrm{MN}$ from the correspondent node $(\mathrm{CN})$ the $\mathrm{HA}$ forwards the packet to the COA of the MN. This whole procedure is implemented with the concept of tunneling and IP-in-IP encapsulation. Whenever there is a change in the COA of the MN it is updated at HA by using location update signaling.

On the other hand when $\mathrm{MN}$ has to send the packets to $\mathrm{CN}$, it can do send it first to FA (if FA-COA is being used by MN) or it can send directly to $\mathrm{CN}$ (if it is using collocated-COA). In this, a very inefficient scenario is developed when $\mathrm{CN}$ and $\mathrm{MN}$ are in close topological proximity and HA is very far away from MN. This scenario is termed as triangular routing. This inefficient triangular routing problem is solved in mobile IPv6 (MIPv6). MIPv6 possesses some inherent field in header which removes triangular routing and provides support for route optimization. There is no need of FA in MIPv6. However when the MN sends packets directly (route optimization), there is disadvantage which is associated with the MIPv6, that the CN must be capable to support mobility-aware operations. Hence although the MIPv6 is solving the triangular routing problem and providing route optimization, it is still showing weakness, when both $\mathrm{CN}$ and $\mathrm{MN}$ are on-the-go. The situation becomes worst when $\mathrm{MN}$ and $\mathrm{CN}$ are moving simultaneously.

Thus we see that the fundamental Mobile IP methods are having the problems in performing real time applications handover. This is because of the longer delays involved in movement detection of $\mathrm{MN}$ and $\mathrm{CN}$, configuration of the new COA, and location update signaling on each network change instance. Some more optimizations are being done in this regard like Fast MIPv6 (FMIPv6) and hierarchical MIPv6 (HMIPv6).

\subsection{Optimizations of MIP}

2.1.1 FMIPv6. FMIPv6 is providing a better freedom for seamless mobility and closer seamless experience. The working of FMIPv6 is very proactive by allowing the MN to fetch the required information about the future subnet in advance before the handover and begins to use next link before getting the location update of the $\mathrm{CN}$ id decided. As shown in figure 2 handover operation preparation is started pro-actively between MN and previous first hop router (FHR) and in between previous FHR and next FHR. Only the context ( access control, QoS profile and header compression) is being transferred between FHRs and new are setup between MN and CN. The complexity is increased at MN and FHRs in FMIPv6.

2.1.2 HMIPv6. HMIPv6 uses the local mobility anchor point (LMAP) to achieve local mobility management as shown in figure 2. In this LMAP works as HA in the local mobility domain. Whenever the MN moves to other subnet in the local mobility domain, the COA belonging to FHR is bind with LMAP which also contain its regional-COA. No change is implemented in the tunnel from LMAP to outside network only the local binding is done. In this way HMIPv6 also reduces the delay to some extant. All these protocols discussed so far, are based on the fundamental Mobile IP methods in which mobility related functionalities are implemented in the MN. When the mobility related functionalities are implemented somewhere in the network entities then a better resource utilization and load balancing can be achieved (because in this case every cell load information is available). IETF devel- oped a new network controlled mobility protocol named as Proxy MIPv6 (PMIPv6). PMIPv6 implements the mobility related functionalities in FHRs and in LMAPs. In this FHR keeps a closer look on the movement of the MN, and whenever there is change in the network it informs LMAP for signaling COA update. All these discussions so far, focuses on the implementation of the interaction between different network entities, i.e. Inter-entity interaction (optimizations at network layer). But handover delays are not only based on inter-entity interaction but also on intra-entity interaction. The delays due to intra-entity interactions are generated because of the latencies induced by the functions. The latencies could be both at link layer and network layer. Like for example the factors for link layer latencies are late detection of link state changes, and lengthy scanning of authentication and association procedures. Similarly at network layer, late detection of the loss of IP connectivity or late movement detection of MN.

\subsection{Mobility Management at Application Layer[12]}

End-to-end mobility management is handled by session initiation protocol (SIP), which is an application layer protocol to perform signaling. SIP has been selected by the Third Generation Partnership Project (3GPP) as the signaling protocol to set up and control real-time multimedia sessions.

\section{IEEE 802.21:MEDIA INDEPENDENT HANDOVER FRAMEWORK [8]}

\subsection{Objectives of IEEE 802.21}

The main objective of this IEEE standard is to facilitate the handover between heterogeneous networks. This standard provides the support for the following:

(1) Vertical handover for both stationary and mobile users

(2) Changing the link according to the speed and bandwidth requirements of an application

(3) Maintaining the service continuity during handover up to a possible extent.

(4) Cooperation of information between $\mathrm{MN}$ and network infrastructure

(5) Available Networks detection

Information stored within network infrastructure is as follows: Neighborhood cell lists, Location of mobile nodes, Higher Layer Service Availability The initiation of handover process is done using measurement reports and triggers provided by the L 2 on the mobile node.

\subsection{Need for Media Independent Handover Framework Standard}

Since there are multiple RATs which are available around the MN. If we implement the mapping of each RAT with the upper layer protocol format, the complexity becomes very high. Let us consider that there are n number of RATs, available around the MN in an overlapped manner. Each RAT is having its own extension. The number of mappings needed for $n$ RATs will be $n(n-1) / 2$. These mappings and their emerging complexity is shown in figure 2 . To overcome this problem, we need one standard which is automatically providing the mappings between various RATs. IEEE 802.21 is doing this for us. It is not only doing this but also helping in choosing appropriate RAT which is most suitable for the MN in the particular mobility scenario. 


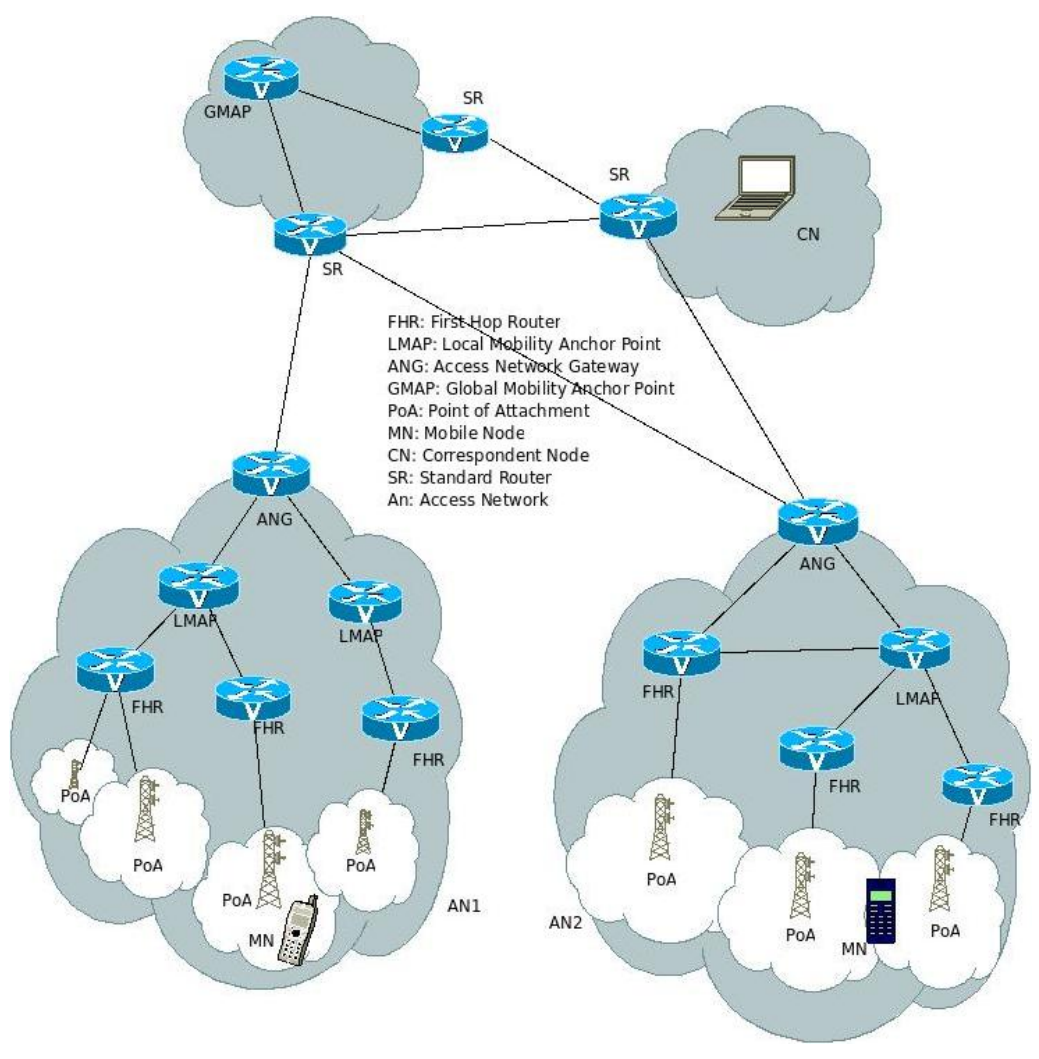

Fig. 2. Mobility Scenario and Mobility Entities in IP Networks

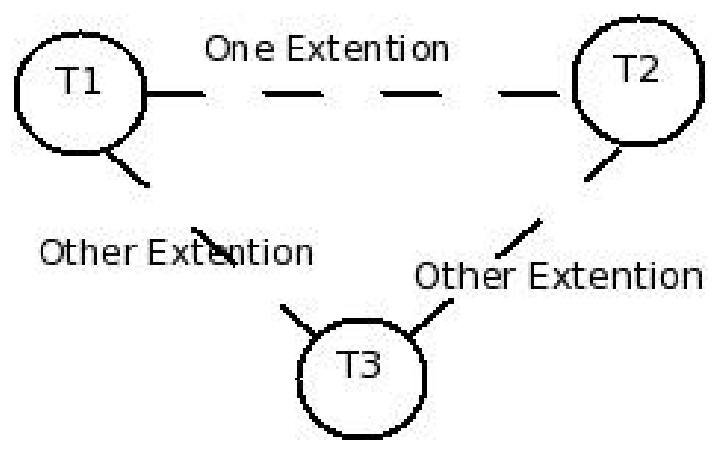

Ti----> RATS

Fig. 3. Mapping Complexity of Multiple RATs

\subsection{Realization of Seamless Mobility Based on IEEE 802.21 Framework}

Figure 5 shows the placement of logical entity based on IEEE 802.21 to realize the seamless mobility in future multi-access Heterogeneous network. As shown in the figure 5 the logical entity MIHF is placed in between layer 2 and layer 3 or upper layers. The figure also shows various service access points (SAPs) towards layer 2 and towards upper layers. On upper layer side, various MIH users (MIP, SIP etc) are residing. There are two types of interfaces which are used by MIHF to provide mobility freedom: one is Media Independent Interfaces and second is Media dependent interfaces. All these interfaces are implemented by using SAPs. There are basic three types of SAPs:
(1) MIH_SAP
(2) MIH_Link_SAP
(3) MIH_NET_SAP 


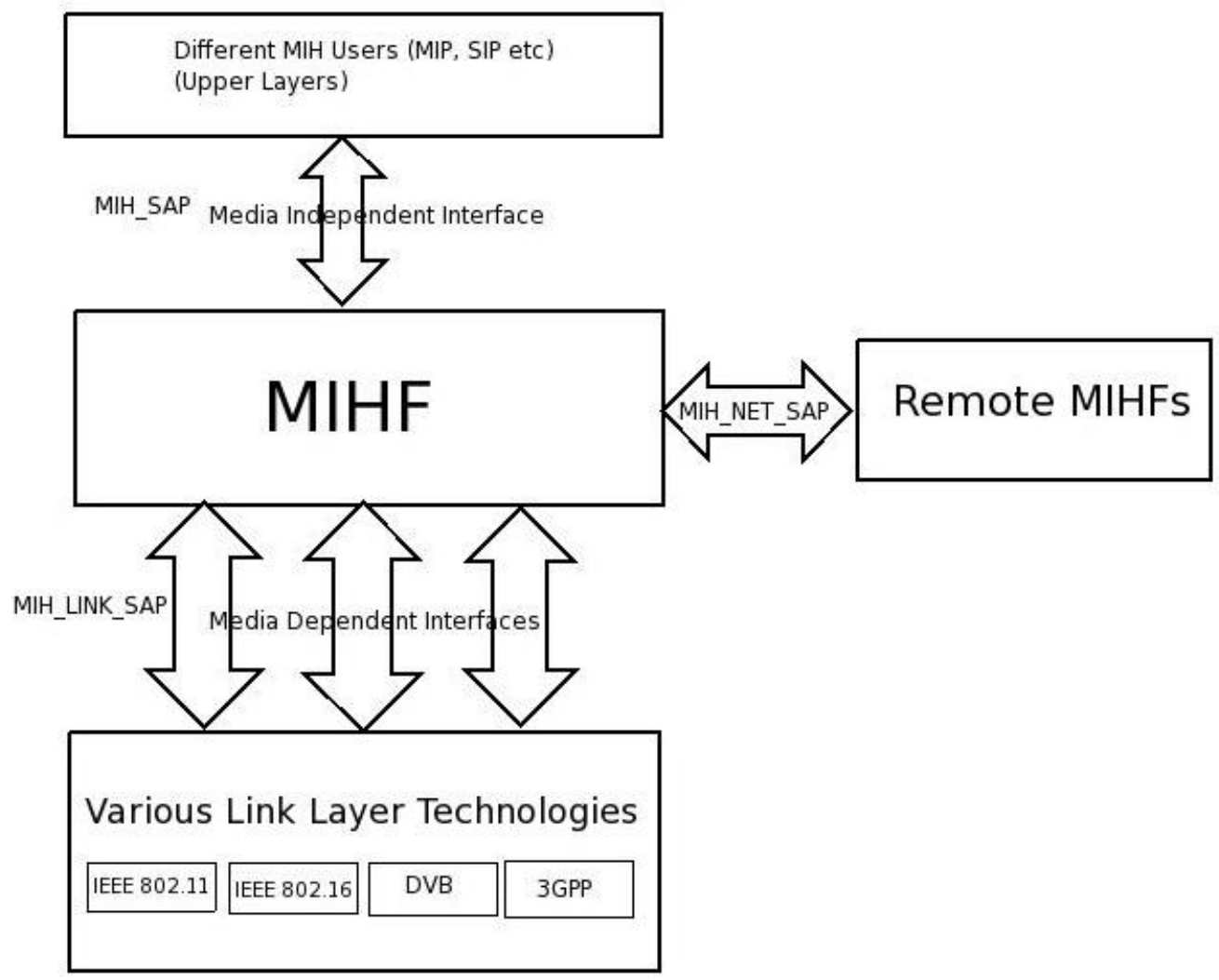

Fig. 4. MIHF and Its Interfaces, Deployment Location and MIH users 7 14

MIH_SAP is media independent interface, while MIH_Link_SAP and MIH_NET_SAP are media dependent interfaces. Some of the media dependent interfaces are as follows:

(1) MLME_SAP: This is the interface between MIHF and the management plane of an IEEE 802.11 (WLAN) network. It is used to send MIH messages between MIHF and local link layer entities and between peer MIHF entities.

(2) C_SAP: This is the interface between MIHF and functions of control plane of IEEE 802.16 (Wi-MAX).

(3) M_SAP: This is the interface between MIHF and functions of management plane of IEEE 802.16 (Wi-MAX).

(4) MSGCF_SAP: This is the interface between MIHF and IEEE 802.11 MAC state machine.

(5) MIH_3GLINK_SAP: This is the interface between MIHF and different protocols of cellular systems.

(6) LSAP: This is the interface between MIHF and logical link control (LLC) of IEEE 802.3 (Ethernet), and IEEE 802.11 (WLAN)

(7) CS_SAP: This is the interface between MIHF and service specific convergence sublayer of IEEE 802.16 (Wi-MAX).

The basic purpose of the IEEE 802.21(MIH) standard is to facilitate handover between heterogeneous networks to provide seamless mobility. The standard supports handover for both mobile as well as stationary users. For mobile users, handover can occur when wireless link conditions change due to MN movement. For stationary users handover can occur environmental changes. The handover process can be initiated by using measurement reports and link layer triggers the handover. The measurement reports can include the metrics such as- signal quality, synchronization time differences and transmission error rates. There are two paradigm for handover initiation, one make-before-break and other is breakbefore-make. MIHF provides three types of service specifications: MIES, MICS and MIIS

3.3.1 Media Independent Event Service (MIES). It detects change in logical link characteristics dynamically such as link status and link quality. It also initiates changes in physical, data link layers. It has two types of events: one is link events which originate from lower layers and proceeds towards upper layers, other is MIH events which are initiated by MIHF.

3.3.2 Media Independent Command Service (MICS). It facilitates controlling of a link state. MICS uses commands for local events and remote events separately. Local events happen within the same protocol stack by local MIHF and remote events happen between local MIHF and remote MIHF subscribed by a remote node.

3.3.3 Media Independent Information Service (MIIS). The MIIS provides the information which is required to perform handover and is linked to the appropriate module for each MIH enabled entity. It gathers the following information which either locally or 
remotely available:

Access Network Related:-

(1) List of Available Networks.

(2) List of Authorized users

(3) Target QoS Parameters for ongoing sessions

(4) Number of Connections per PoA

(5) Load Factor(Uplink or Downlink)

(6) Available Bandwidth

MN Related:-

(1) Detected Networks

(2) Parameters for context Transfer

(3) PHY Parameters

(4) QoS Parameters for the Network

(5) Measured QoS Parameters Experienced For the Network

MIHF uses the triggers from link layer to support handover initiation and handover preparation. Handover initiation consists three phases:

(i) Network Discovery

(ii) Network Selection

(iii) Handover Negotiation. While the handover preparation has two steps: (i) Lower and upper layer connectivity (ii) Resource Reservation

MN can work with WLAN, WPAN, DVB, 3GPP and some other unlicensed mobility interfaces and uses the facilities provided by the MIHF to achieve seamless mobility. The MIH users of MIHF at the MN side are MIP, mobile stream control transmission protocol (mSCTP), service initiation protocol (SIP) respectively at network, transport and application layers.

\section{NETWORK SELECTION BASED ON SERVICE ZONE[13]}

The authors have described the vertical handover for macro mobility by proposing VHO technique for selection of networks based on network service zone using GPS among multiple available networks for seamless network connectivity. They have assumed that the MN is assisted by a GPS server to track the real time location. This is depicted in figure 3. In [13], the network selection and switching technique is proposed by considering the user preferences and network performance without using network hardware dependent metrics (eg., RF strength, RF link quality, and so on) and is independent of network metrics such as RTT (Round Trip Time) and PLR (Packet Loss Rate).

\section{VERTICAL HANDOVER MANAGEMENT}

Handover management handles the movement of MN by keeping the connection active while changing the PoA (access point, base station).

\subsection{Vertical Handover}

The process of changing the PoA by MN across the networks is known as vertical handover. It is defined as the switch between two different RAT or the switch between two different radio access networks operating with the same technology if the IP address of the $\mathrm{MN}$ changes as a result of the handover. Vertical handover provides media independence using IEEE 802.21, therefore it is also known as media independent handover. We have simulated the media independent handover between WLAN and UMTS (3G cellular technology) in [14].

\subsection{Need of Vertical Handover}

Now the MN is being equipped with multiple network interfaces. These multiple network interfaces can help extend the range of the wireless networks. Vertical handover is needed in this extended wireless network of overlapped coverage of various types of RATs. Also, VHO provides flexibility and cost effective usage of overlapped RATs coverage. VHO is also being used to achieve load balancing.

\subsection{Vertical Handover Performance Issues}

VHO is the main barrier to be crossed in the way of achieving seamless mobility. There are many issues which derives the performance of VHO such as discovering the available RATs around the multiinterface enabled $\mathrm{MN}$, detection of received signal strength (RSS) of each RAT, selecting the optimal RAT according to the user preferences, RAT is preferred by user, determining the gain of handing over to the preferred RAT, determining the exact time of VHO and execution of VHO.

VHO involves the following decision factors: Decision Criteria, Decision Policies, Decision Algorithms, Decision Control Schemes

\subsection{VHO Decision Criteria}

In [2], following VHO decision criteria is described:

(1) User Preferences

(2) Network Conditions (Received Signal Strength (RSS), QoS Parameters, Mobility Pattern)

(3) Application Requirements (Velocity of MN)

(4) MN Capabilities (Processing Power, Battery Status, Interface Support)

\subsection{VHO Decision Algorithms|2]}

(1) Function-based algorithms

(2) User-centric algorithms

(3) Multiple attributes based algorithms

(4) Fuzzy Logic and Neural Network based algorithms

(5) Context aware algorithms

5.5.1 Service History based VHO Algorithms [11]. In [11], service history based VHO algorithm is used to reduce un-necessary and frequent handovers. Seamless handover is an important issue either it is achieved using horizontal or vertical handover algorithms. Each candidate network is evaluated for multiple attribute such as available bandwidth, delay, data rate and cost. Frequent handover occurs because of variations in these parameters. Disconnection probability also tends towards high because of change in these parameters. Following decision criteria is used in service history based $\mathrm{VHO}$ algorithm:

DecisionCriteria $=F($ availablebandwidth, delay, datarate, $\cos t)$

In [11], an objective function is proposed which is specifying two parameters from service history information: first parameter is cumulative service time since the last handover at a particular serving network, second parameter is time duration since the last handover blocking from a particular network. Both these parameters were optimized so that frequent handover (ping ponging) and handover blocking is avoided. 


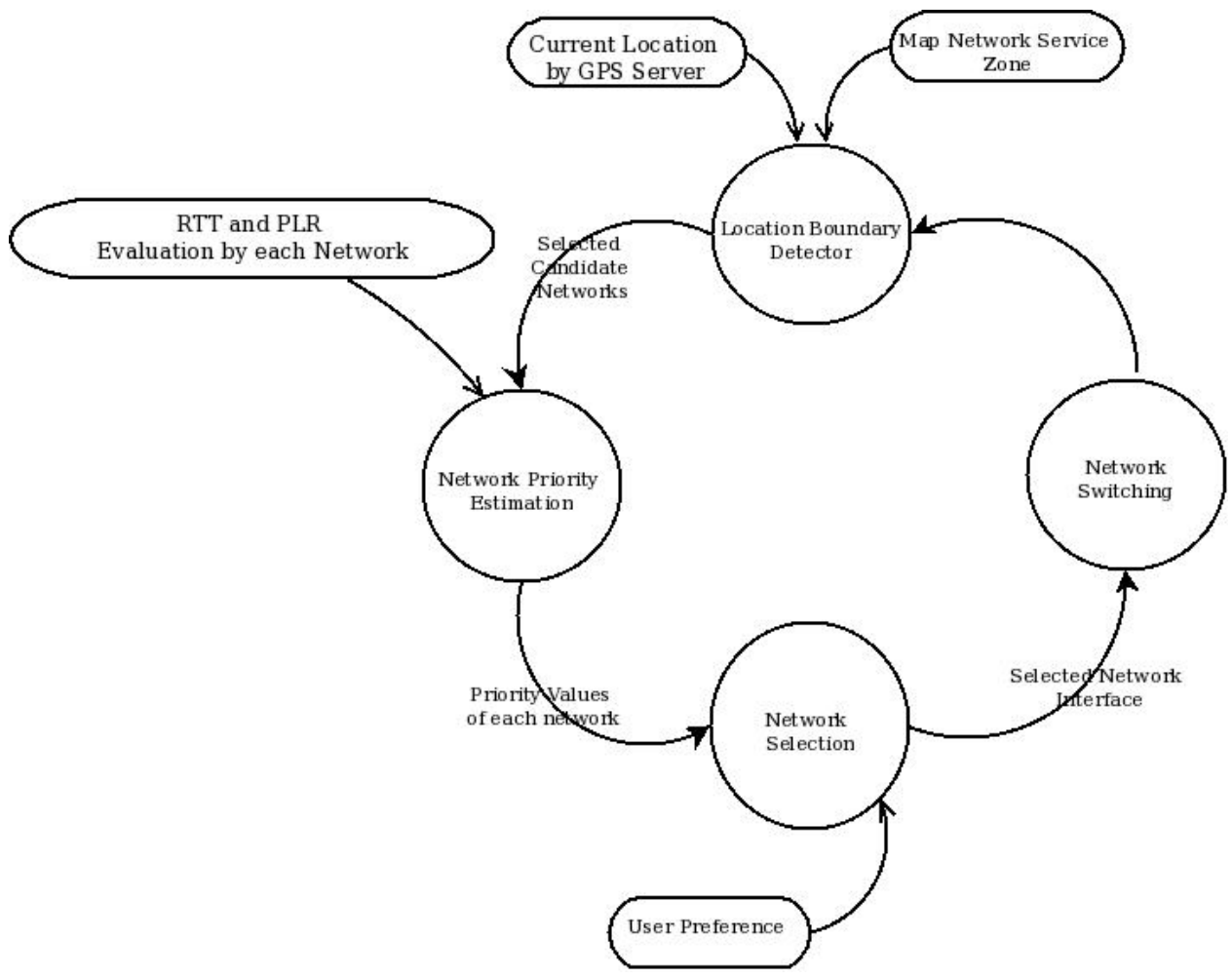

Fig. 5. Typical Stages of Vertical Handover for seamless macromobility

\subsection{Vertical Mobility Management Architectures}

A comprehensive survey of vertical mobility management architectures is provided in [15]. In this it is described that protocols for mobility, fair comparision of different solutions at each layer and their various associated problems, design and performance evaluation of different handoff procedures are the current architectural issues which are point of concern among researchers.

\section{SIMULATION \& RESULTS}

\subsection{Simulation of MIH for IEEE 802.11 and UMTS}

We simulated a heterogeneous network which contains two types of networks: UMTS and IEEE 802.11 (WLAN). Universal Mobile Telecommunications System is a $3 \mathrm{G}$ cellular technology, while Wireless Local Area Network is member of IEEE 802 project. We have simulated the vertical handover between these two by using NS-2.29 version of NS and mobility extensions package provided by National Institute of Standards and Technology(NIST).

6.1.1 Simulation Scenario. The considered simulation scenario to draw simulation results, has an assumption that the coverage area of UMTS cell is the entire simulation map. The WLAN cell is located inside the UMTS cell. The UMTS cell has a base station(BS) and the WLAN cell has access point(AP). Initially, the multi-interface equipped MN is connected to the UMTS BS. The preference is always given to the WLAN interface as soon as it is available. The simulation scenario is shown in the figure 3 . It is clear from figure 4 that $\mathrm{MN}$ performs two handovers, one handover when it leaves UMTS cell and joins WLAN cell. and other han- dover is when it leaves WLAN cell and the traffic is redirected to UMTS BS.

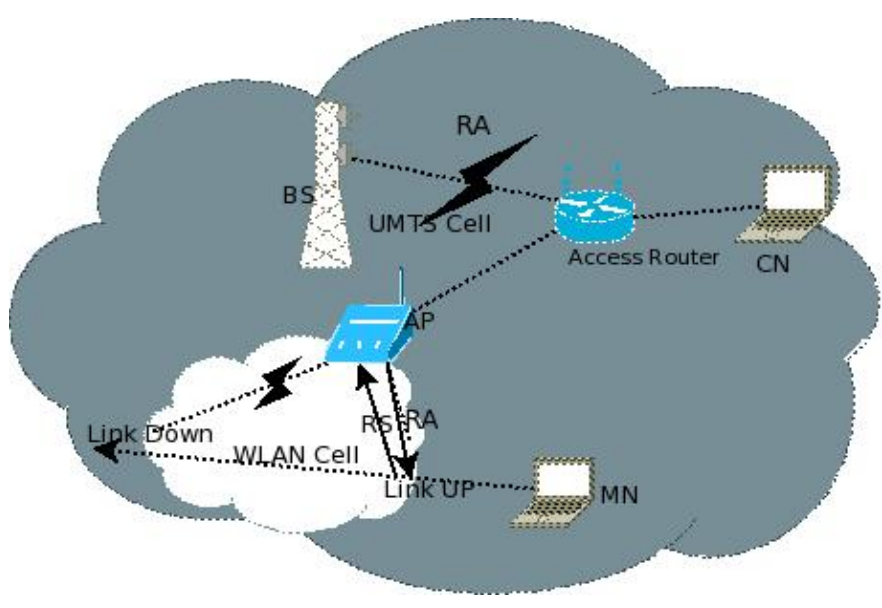

Fig. 6. MIH Simulation Scenario

6.1.2 Simulation Parameters. Tables 2, 3, 4 \& 5 show the parameters for simulation of MIH to and from WLAN and UMTS. We have considered straight line mobility model. The application traffic type for MN is UDP with packet size 500 bytes and packet interval time is $0.02 \mathrm{~s}$. 
Table 2.

Topology

\begin{tabular}{|l|l|l|}
\hline S.No & Parameter & Value \\
\hline 1. & UMTS Cell Coverage & Entire Simulation Map \\
\hline 2. & WLAN Cell Coverage & $20 \mathrm{~m}$ \\
\hline 3. & Propagation Delay CN-MN & 0.09 s for RTT + Mac access delay \\
\hline 4. & Nodes in WLAN Hotspots & 0 To 20 \\
\hline
\end{tabular}

Table 3.

Parameters_of_the_Router

\begin{tabular}{|l|l|l|}
\hline S.No & Parameter & Value \\
\hline 1. & MIN_RA_DELAY & 200s \\
\hline 2. & MAX_RA_DELAY & 3XMIN_RA_DELAY \\
\hline 3. & Router Lifetime & 3XMIN_RA_DELAY \\
\hline 4. & MIN_DELAY_Between_RA & 0 \\
\hline
\end{tabular}

Table 4.

MAC_Parameters

\begin{tabular}{|l|l|l|}
\hline S.No & Parameter & Value \\
\hline 1. & WLAN beacon interval & $0.1 \mathrm{~s}$ \\
\hline 2. & Default Scanning Mode & Passive \\
\hline 3. & MinChannelTime & $0.02 \mathrm{~s}$ \\
\hline 4. & MaxChannelTime & $0.06 \mathrm{~s}$ \\
\hline 5. & ProbeDelay & $0.002 \mathrm{~s}$ \\
\hline
\end{tabular}

\section{CONCLUSIONS \& FUTURE WORK}

Mobility solutions are are required at almost all layers of layered architecture. We have described the mobility solutions at application layer and network layer. The main obstacle in achieving seamless mobility is vertical handover and its related issues and challenges. In this paper we have presented a comprehensive description of issues and challenges to achieve seamless mobility in a heterogeneous wireless networks. The main issues to achieve seamless mobility are discovery of available RATs, optimal selection of user preferred RAT, deciding exact time of initiating and completing VHO, VHO algorithms. As a case study, we simulated vertical handover between IEEE 802.11 (WLAN) and UMTS(Cellular Technology) and presented our observations using NS2. We are still working on other issues to achieve the seamless mobility like to estimate exact time to perform vertical handover, RAT detection, developing multi-parameter cost function.

\section{Acknowledgments}

We wish to thank the anonymous reviewers for their valuable suggestions. Our sincere thanks to Dr. Avdhesh Kumar (Head, CSE and CIS) Galgotias University and our friends for their support during this work.

\section{REFERENCES}

[1] N. Golmie, Seamless Mobility: Are We There Yet? IEEE Wireless Communications August 2009.

[2] Meriem Kassar et al., An Overview of Vertical Handover Decision Strategies in Heterogeneous Wireless Networks IEEE Elsevier Jounal on Computer Communications Science Direct, January 2008.
[3] Lambros Sarakis et al., Seamless Service provision For Multi Heterogeneous Access IEEE Wireless Communications, October 2009.

Table 5.

UMTS_Parameters

\begin{tabular}{|l|l|l|}
\hline S.No & Parameter & Value \\
\hline 1. & Transport Channel & Dedicated Channel \\
\hline 2. & LinkLayerType & UMTS/RLC/AM \\
\hline 3. & DownLinkBW & $384 \mathrm{kbps}$ \\
\hline 4. & UpLinkBW & $384 \mathrm{kbps}$ \\
\hline
\end{tabular}

[4] Jonathen Rodriguez et al., A Middle-Ware Architecture Supporting Seamless and Secure Multimedia Services Across an Inter-technology Radio Access Network IEEE Wireless Communications, October 2009.

[5] Christian M. Mueller et. al., Realization Aspects of MultiRadio Management Based on IEEE 802 .21 WWIC 2009, LNCS 5546, pp. 109-120 2009 Springer-Verlag Berlin Heidelberg 2009.

[6] Vivek Gupta, Mobility Using IEEE 802.21 in a Heterogeneous IEEE 802.16/802.11- Based, IMT-Advanced (4G) Network IEEE Wireless Communications, April 2008 .

[7] Kenichi Taniuchi et. al., IEEE 802.21: Media Independent Handover: Features, Applicability, and Realization IEEE Communications Magazine, January 2009.

[8] IEEE 802.21-2008, IEEE Standard for Local and Metropolitan Area Networks Media Independent Handover Services, Jan. 2009.

[9] Dave Cavalcanti and Dherma Agarwal, Issues in Integrating Cellular Networks, WLANs, and MANETs: A Futuristic Heterogeneous Wireless Network IEEE Wireless Communications, June 2005.

[10] Antonio De La Oliva, et. al., An overview of IEEE 802.21: Media Independent Handover Services IEEE Wireless Communications, August 2008

[11] Taehoon Kim, Sang-wook Han, and Youngnam Han A QoSaware Vertical Handover algorithm based on Service History Information IEEE Communications Letters Vol14, No.6 June 2010

[12] STEFANO SALSANO AND ANDREA POLIDORO SIPBASED MOBILITY MANAGEMENT IN NEXT GENERATION NETWORKS IEEE Wireless Communications April 2008

[13] Jinhee Park AND Jinwook Chung Network Selection based on Network Service Zone for Macro Mobility ICIS November 2009

[14] Vimal Kumar, Neeraj Tyagi Media Independent Handover for Seamless Mobility in IEEE 802.11 and UMTS based on IEEE 802.21 IEEE ICSIT July 2010

[15] Stenio Fernandes, Ahmed Karmouch Vertical Mobility Management Architectures in Wireless Networks: A Comprehensive Survey and Future Directions IEEE COMMUNICATIONS SURVEYS \& TUTORIALS, VOL. 14, NO. 1, FIRST QUARTER 2012 